\title{
Non-Resonant Vibrational Energy Transfer on Metal Nanoparticle/Liquid Interface
}

Jiebo Li ${ }^{1,2}$, Hailong $\mathrm{Chen}^{2}$, Andrea Miranda ${ }^{2}$, Kaijun Yuan $^{3}$, Yuneng Shen ${ }^{3}$, Bo Jiang ${ }^{2,3}$, Yajing Chen $^{3}$, Yufan Zhang ${ }^{2}$, Xunmin Guo ${ }^{2}$, Junrong Zheng ${ }^{1,2 *}$

${ }^{1}$ College of Chemistry and Molecular Engineering, Beijing National Laboratory for Molecular Sciences, Peking University, Beijing 100871, China

${ }^{2}$ Department of Chemistry, Rice University, Houston, Texas, USA

${ }^{3}$ State Key Laboratory of Molecular Reaction Dynamics, Dalian Institute of the Chemical Physics, Chinese Academy of Sciences, Dalian, Liaoning, China

*To whom correspondence should be addressed: zhengjunrong@gmail.com

\section{This file includes:}

Figure S1 to S6

Table S1 and S2

Supporting Information text

References 


\section{List of all the vibrational excitation population of Atop $\mathrm{CO}$ on first and second layer}

Layer 1:

$\frac{d\left[\mathrm{CO}^{11}\right]_{\text {Atop }}}{d t}=$

$-k_{11 \text { Atop-11Bridge }}\left[C O^{11}\right]_{\text {Atop }}+k_{11 \text { Bridge-11Atop }}\left[\mathrm{CO}^{11}\right]_{\text {Bridge }}-k_{11 \text { Atop-12Bridge }}\left[\mathrm{CO}^{11}\right]_{\text {Atop }}+k_{12 \text { Bridge-11Atop }}\left[C \mathrm{CO}^{12}\right]_{\text {Bridge }}$

$-k_{11 \text { Atop-13Bridge }}\left[C O^{11}\right]_{\text {Atop }}+k_{13 \text { Bridge-11Atop }}\left[\mathrm{CO}^{13}\right]_{\text {Bridge }}-k_{11 \text { Atop-21Bridge }}\left[C O^{11}\right]_{\text {Atop }}+k_{21 \text { Bridge-11Atop }}\left[\mathrm{CO}^{21}\right]_{\text {Bridge }}$

$-k_{11 \text { Atop-22Bridge }}\left[\mathrm{CO}^{11}\right]_{\text {Atop }}+k_{22 \text { Bridge-11Atop }}\left[\mathrm{CO}^{22}\right]_{\text {Bridge }}-k_{11 \text { Atop-23Bridge }}\left[\mathrm{CO}^{11}\right]_{\text {Atop }}+k_{23 \text { Bridge-11Atop }}\left[\mathrm{CO}^{23}\right]_{\text {Bridge }}$

$-k_{11 \text { Atop-31Bridge }}\left[\mathrm{CO}^{11}\right]_{\text {Atop }}+k_{31 \text { Bridge-11Atop }}\left[\mathrm{CO}^{31}\right]_{\text {Bridge }}-k_{\text {Atop }}\left[\mathrm{CO}^{11}\right]_{\text {Atop }}$

Eq. S1

$$
\begin{aligned}
& \frac{d\left[\mathrm{CO}^{21}\right]_{\text {Atop }}}{d t}= \\
& -k_{21 \text { Atop-11Bridge }}\left[\mathrm{CO}^{21}\right]_{\text {Atop }}+k_{11 \text { Bridge-21 Atop }}\left[\mathrm{CO}^{11}\right]_{\text {Bridge }} \\
& -k_{21 \text { Atop-12Bridge }}\left[\mathrm{CO}^{21}\right]_{\text {Atop }}+k_{12 \text { Bridge-21Atop }}\left[\mathrm{CO}^{12}\right]_{\text {Bridge }} \\
& -k_{21 \text { Atop-13Bridge }}\left[\mathrm{CO}^{21}\right]_{\text {Atop }}+k_{13 \text { Bridge-21Atop }}\left[\mathrm{CO}^{13}\right]_{\text {Bridge }} \\
& -k_{21 \text { Atop-21Bridge }}\left[\mathrm{CO}^{21}\right]_{\text {Atop }}+k_{21 \text { Bridge-21 Atop }}\left[\mathrm{CO}^{21}\right]_{\text {Bridge }} \\
& -k_{21 \text { Atop-22Bridge }}\left[\mathrm{CO}^{21}\right]_{\text {Atop }}-k_{22 \text { Bridge-21 Atop }}\left[\mathrm{CO}^{22}\right]_{\text {Bridge }} \\
& -k_{21 \text { Atop-23Bridge }}\left[\mathrm{CO}^{21}\right]_{\text {Atop }}+k_{23 \text { Bridge-21Atop }}\left[\mathrm{CO}^{23}\right]_{\text {Bridge }} \\
& -k_{21 \text { Atop-31Bridge }}\left[\mathrm{CO}^{21}\right]_{\text {Atop }}+k_{31 \text { Bridge-21Atop }}\left[\mathrm{CO}^{31}\right]_{\text {Bridge }} \\
& -k_{21 \text { Atop-32Bridge }}\left[\mathrm{CO}^{21}\right]_{\text {Atop }}+k_{32 \text { Bridge-21 Atop }}\left[\mathrm{CO}^{32}\right]_{\text {Bridge }} \\
& -k_{21 \text { Atop-33Bridge }}\left[\mathrm{CO}^{21}\right]_{\text {Atop }}+k_{33 \text { Bridge-21Atop }}\left[\mathrm{CO}^{33}\right]_{\text {Bridge }} \\
& -k_{\text {Atop }}\left[C O^{21}\right]_{\text {Atop }}
\end{aligned}
$$

Eq. S2

Layer 2 


$$
\begin{aligned}
& \frac{d\left[\mathrm{CO}^{31}\right]_{\text {Atop }}}{d t}= \\
& -k_{31 \text { Atop-21Bridge }}\left[\mathrm{CO}^{31}\right]_{\text {Atop }}+k_{21 \text { Bridge-31 Atop }}\left[\mathrm{CO}^{21}\right]_{\text {Bridge }} \\
& -k_{31 \text { Atop-22Bridge }}\left[\mathrm{CO}^{31}\right]_{\text {Atop }}+k_{22 \text { Bridge-31 Atop }}\left[\mathrm{CO}^{22}\right]_{\text {Bridge }} \\
& -k_{31 \text { Atop-23Bridge }}\left[\mathrm{CO}^{31}\right]_{\text {Atop }}+k_{23 \text { Bridge-31Atop }}\left[\mathrm{CO}^{23}\right]_{\text {Bridge }} \\
& -k_{31 \text { Atop-31 Bridge }}\left[\mathrm{CO}^{31}\right]_{\text {Atop }}+k_{31 \text { Bridge-31 Atop }}\left[\mathrm{CO}^{31}\right]_{\text {Bridge }} \\
& -k_{31 \text { Atop-32Bridge }}\left[\mathrm{CO}^{31}\right]_{\text {Atop }}+k_{32 \text { Bridge-31 Atop }}\left[\mathrm{CO}^{32}\right]_{\text {Bridge }} \\
& -k_{31 \text { Atop-33Bridge }}\left[\mathrm{CO}^{31}\right]_{\text {Atop }}+k_{33 \text { Bridge-31 Atop }}\left[\mathrm{CO}^{33}\right]_{\text {Bridge }} \\
& -k_{31 \text { Atop-41Bridge }}\left[C O^{31}\right]_{\text {Atop }}+k_{41 \text { Bridge-31 Atop }}\left[\mathrm{CO}^{41}\right]_{\text {Bridge }} \\
& -k_{31 \text { Atop-42Bridge }}\left[\mathrm{CO}^{31}\right]_{\text {Atop }}+k_{42 \text { Bridge-31 Atop }}\left[\mathrm{CO}^{42}\right]_{\text {Bridge }} \\
& -k_{31 \text { Atop-43Bridge }}\left[C O^{31}\right]_{\text {Atop }}+k_{43 \text { Bridge-31 Atop }}\left[\mathrm{CO}^{43}\right]_{\text {Bridge }} \\
& -k_{\text {Attop }}\left[C O^{31}\right]_{\text {Atop }}
\end{aligned}
$$

Eq. S3

The resonant energy transfer term of $[\mathrm{CO}]$ Atop would not be considered in this expression. Thus, all the parameters in the above equation Eq.S1 are expressed by $k_{\text {llAtop-llBridge. The values are }}$ listed in Table S1.

$$
\begin{aligned}
& k_{11 \text { Atop-11 Bridge }}=1 k_{11 \text { Atop-11Bridge }} ; k_{11 \text { Atop-12Bridge }}=1.34 k_{11 \text { Atop-11 Bridge }} ; k_{11 \text { Atop-13Bridge }}=0.10 k_{11 \text { Atop-11Bridge }} ; \\
& k_{11 \text { Atop-21Bridge }}=0.14 k_{11 \text { atop-11Bridge }} ; k_{11 \text { Atop-22Bridge }}=0.06 k_{11 \text { Atop-11Bridge }} ; k_{11 \text { Atop-23Bridge }}=0.02 k_{11 \text { Atop-11Bridge }} ; \\
& k_{11 \text { Atop-31Bridge }}=0.02 k_{11 \text { Atop-11Bridge }}
\end{aligned}
$$

Rewriting the eq. S1, we would obtain the equation S4 as following:

$$
\frac{d\left[\mathrm{CO}^{11}\right]_{\text {Atop }}}{d t}=-2.68 k_{11 \text { Atop -11 Bridge }}\left[\mathrm{CO}^{11}\right]_{\text {Atop }}+2.68 k_{11 \text { Bridge-11 Atop }}\left[\mathrm{CO}^{11}\right]_{\text {Bridge }}-k_{\text {Atop }}\left[\mathrm{CO}^{11}\right]_{\text {Atop }}
$$

Using the same method, it is straightforward to calculate the dynamics of the excitation population of $\left[\mathrm{CO}^{21}\right]$ and $\left[\mathrm{CO}^{31}\right]$ with $k_{\text {llatop-llBridge }}$ in equations $\mathrm{S} 5$ and $\mathrm{S} 6$.

$$
\frac{d\left[\mathrm{CO}^{21}\right]_{\text {Atop }}}{d t}=-3.75 k_{11 \text { Atop }-11 \text { Bridge }}\left[\mathrm{CO}^{21}\right]_{\text {Atop }}+3.75 k_{11 \text { Bridge-11 1Atop }}\left[\mathrm{CO}^{21}\right]_{\text {Bridge }}-k_{\text {Atop }}\left[\mathrm{CO}^{21}\right]_{\text {Atop }}(\mathrm{Eq}
$$


$\frac{d\left[\mathrm{CO}^{31}\right]_{\text {Atop }}}{d t}=-3.84 k_{11 \text { Atop-11Bridge }}\left[\mathrm{CO}^{31}\right]_{\text {Atop }}+3.84 k_{11 \text { Bridge-11Atop }}\left[\mathrm{CO}^{31}\right]_{\text {Bridge }}-k_{\text {Atop }}\left[\mathrm{CO}^{31}\right]_{\text {Atop }}$ (Eq.S6)

We roughly assumed that the atop site COs on all five layers would have an equal possibility of excitation. (The double excitation on one particle is difficult. Based on our experimental condition, the sample $\mathrm{CO}$ (at atop site) concentration is $\sim 5 \mathrm{mM}$. The optical pump beam pulse at this wavelength is $10 \mu \mathrm{J}$. The beam diameter is around $300 \mu \mathrm{m}$. Thus, the photon density is around $10^{-10} \mathrm{M}$, which is much lower than $\mathrm{CO}$ concentration. Thus, we only consider the single excited particle case. A simple estimation is given for the $1 \%$ excitation probability. For each site, $1 \%$ excitation means $15 \%$ for one $\mathrm{CO}$ excited on one site of one particle. The possibility for two or more $\mathrm{CO}$ molecules on one particle to be excited is $0.01^{2} \times C_{15}^{2}+0.01^{3} \times C_{15}^{3}+0.01^{4} \times C_{15}^{4}+\ldots+0.01^{15} \times C_{15}^{15}$ which is about $1 \%$. The ratio of double excitation over single excitation is $1 / 15$. We also assumed CO molecules on the top (layer 1) and bottom layers (layer 5) are equivalent for energy dissipation pathways. Thus, $\left[\mathrm{CO}^{11}\right]_{\text {Atop }}$ could represent $\mathrm{CO}$ on all 6 molecules on both the top and bottom layer. Also, according to the molecular structure, $\mathrm{CO}$ molecules on layer 2 and layer 4 are subject to the same assumption. Here, $\left[\mathrm{CO}^{21}\right]_{\text {Atop }}$ is used to describe all $\mathrm{CO}$ dynamics on layers 2 and 4 . Therefore, presenting all the possible excited populations of the atop $\mathrm{CO}$ molecules, we obtain the energy transfer rate $k_{\text {Atop-Bridge }}$ from atop $\mathrm{CO}$ to the nearby bridge CO $k_{\text {llAtop-11Bridge }}$ as following:

$$
\begin{aligned}
& 6 \frac{d\left[\mathrm{CO}^{11}\right]_{\text {Atop }}}{d t}+6 \frac{d\left[\mathrm{CO}^{21}\right]_{\text {Atop }}}{d t}+3 \frac{d\left[\mathrm{CO}^{31}\right]_{\text {Atop }}}{d t} \\
& =-49.5 k_{11 \text { Atop-11Bridge }}\left[C O^{31}\right]_{\text {Atop }}+49.5 k_{11 \text { Bridge-31 Atop }}\left[\mathrm{CO}^{21}\right]_{\text {Bridge }}-15 k_{\text {Atop }}\left[C O^{31}\right]_{\text {Atop }}
\end{aligned}
$$

The final expression of CO site is following:

$$
\frac{d[\mathrm{CO}]_{\text {Atop }}}{d t}=-3.3 k_{11 \text { Atop-11Bridge }}[C O]_{\text {Atop }}+3.3 k_{11 \text { Bridge-11Atop }}[\mathrm{CO}]_{\text {Bridge }}-k_{\text {Atop }}[C O]_{\text {Atop }}
$$


In this calculation in Table S1, all rates calculated based on the dipole-dipole coupling. Here, we also discuss the mechanical coupling contribution. If the mechanical coupling are dominated, the

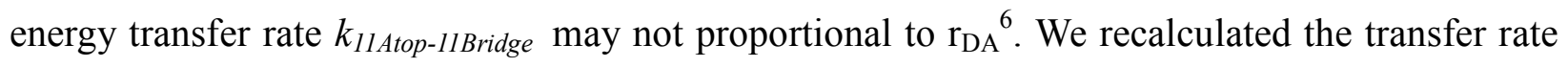

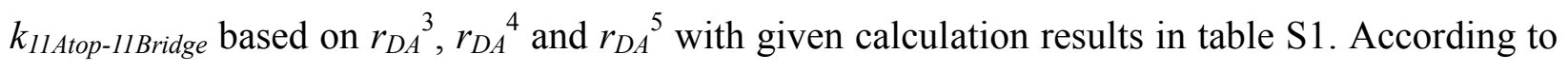
the results showed in table $\mathrm{S} 1$, the energy transfer rate $k_{\text {llAtop-11Bridge }}$ is ranging from $1 / 714 \mathrm{ps}^{-1}$, $1 / 620 \mathrm{ps}^{-1}$, to $1 / 518 \mathrm{ps}^{-1}$ based on the experimental results. According to equation 4 , if coupling strength is stronger, the transfer rate should be much faster than $400 \mathrm{ps}^{-1}$. Therefore, energy transfer mechanism is not dipole-dipole coupling $\left(\mathrm{r}_{\mathrm{DA}}{ }^{6}\right)$, the calculated results would not be consistent with experimental results. 


\section{Fitting the energy transfer kinetic model}

The fiting parameters in Fig 3 are:

$$
\begin{aligned}
& k_{C O_{\text {Atop }} \text { fast }}=1 / 10.6\left(\mathrm{ps}^{-1}\right) ; k_{C O_{\text {Atop }} \text { low }}=1 / 75.0\left(\mathrm{ps}^{-1}\right) ; k_{C O_{\text {Bridge }} \text { slow }}=1 / 42.0\left(\mathrm{ps}^{-1}\right) ; \\
& k_{C O_{\text {Atop }} \rightarrow C O_{\text {Bridge }}}=1 / 180\left(\mathrm{ps}^{-1}\right) ; \mathrm{D}=0.40
\end{aligned}
$$

with pre-factors of the subgroups and offset of the bi-exponential

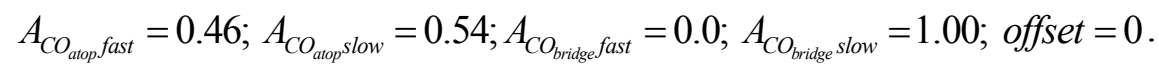

We find that a bi-exponential can better describe the decay of the atop $\mathrm{CO}$, and therefore used the bi-exponential model $^{1}$ described in our previous works.
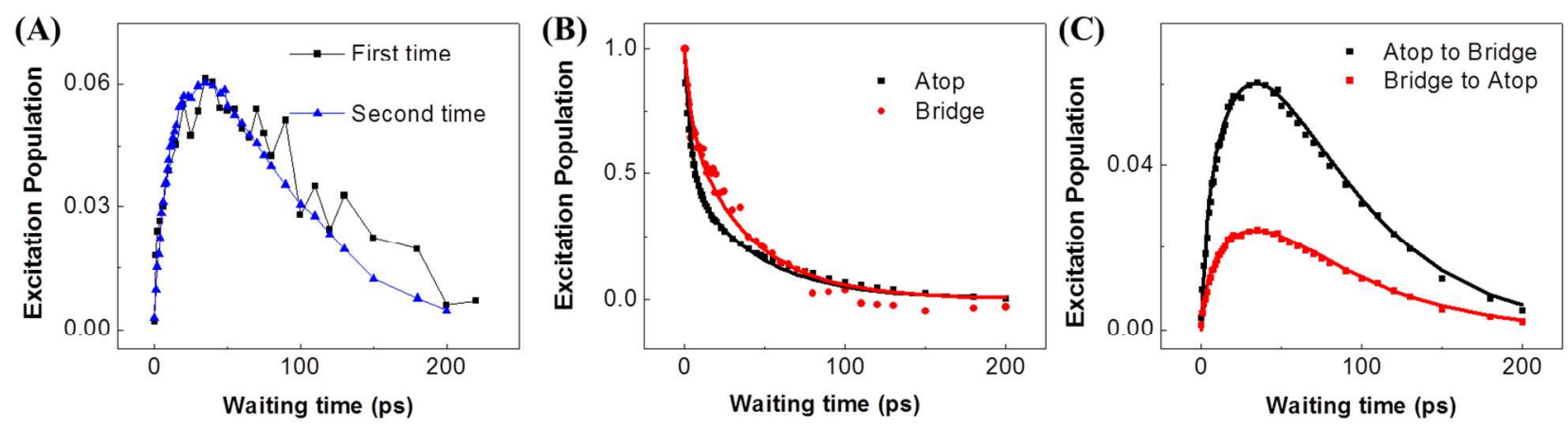

Fig S1 Vibrational excitation populations and kinetic calculations. (A) Comparison of first trail and second trial cross peaks data. (B) Normalized intensity of peak 1 (black) and 3 (red) of second trial. (C) Normalized intensity of peak 5 (black) and 7 (red) of second trail. Dots of $B \& C$ are experimental data, and lines are calculations.

We measured the sample in the second trail to get more smooth energy transfer data but bad energy relaxation data. As shown in Fig S1 (A), the energy transfer data was consistent. But the energy relaxation data was quite difficult to measure precisely in short time because the sample was not stable with light.

The fiting parameters in second trial of Fig 1B\&C are: 


$$
\begin{aligned}
& k_{C O_{\text {Atop }} \text { fast }}=1 / 4.0\left(\mathrm{ps}^{-1}\right) ; k_{C O_{\text {Atop }} \text { Slow }}=1 / 70.0\left(\mathrm{ps}^{-1}\right) ; k_{C O_{\text {Bridege }} \text { fast }}=4.5\left(\mathrm{ps}^{-1}\right) ; k_{C O_{\text {Bridge }} \text { slow }}=1 / 42.0\left(\mathrm{ps}^{-1}\right) ; \\
& k_{C O_{\text {Atop }} \rightarrow C O_{\text {Bridge }}}=1 / 100\left(\mathrm{ps}^{-1}\right) ; \mathrm{D}=0.40
\end{aligned}
$$

with pre-factors of the subgroups and offset of the bi-exponential

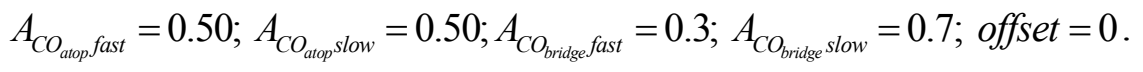

Therefore, we take the final rate of energy transfer from atop $\mathrm{CO}$ to bridge $\mathrm{CO}$ $\left(\frac{1}{k_{\text {Atop } \rightarrow \text { Bridge }}}=140 \pm 40 p s\right)$ as the average rates of the two trials. 


\section{Transition dipole of Atop CO calculation}

The expression for dipole strength is reported ${ }^{2}$ as

$$
\mu^{2}=9.186 \times 10^{-3} n_{(3)} \int[\varepsilon(\tilde{v}) / \tilde{v}] d \tilde{v} \quad \text { Eq. S9 }
$$

where $\mu$ is in units of Debye, $\tilde{v}$ is the wavenumber in $\mathrm{cm}^{-1}, \varepsilon(\tilde{v})$ is the molar decadic extinction coefficient in $\mathrm{L} /(\mathrm{mol} \cdot \mathrm{cm})$ at wavenumber $\varepsilon(\tilde{v})$, and $n_{(3)}$ which denotes the propagation effect is the refractive index of the media.

According to this equation, the transition dipole of $\mathrm{CO}$ on atop site is calculated as following:

$\mu^{2}=9.186 \times 10^{-3} n_{(3)} \int[\varepsilon(\tilde{v}) / \tilde{v}] d \tilde{v}$

$=9.186 \times 10^{-3} n_{(3)} \int\left[\frac{A(\tilde{v}) / \tilde{v}}{C l}\right] d \tilde{v}$

$=9.186 \times 10^{-3} \times 1.430 \times \frac{2.49 \times 10^{-4}}{0.005 \mathrm{~mol} / \mathrm{L} \times 40 \times 10^{-4} \mathrm{~cm}}\left(D^{2}\right)$

$=0.135\left(D^{2}\right)$

$\mu=0.36 D$

$A(\tilde{v})=2.49 \times 10^{-4}$ is integrated from Figure $\mathrm{S} 2$.
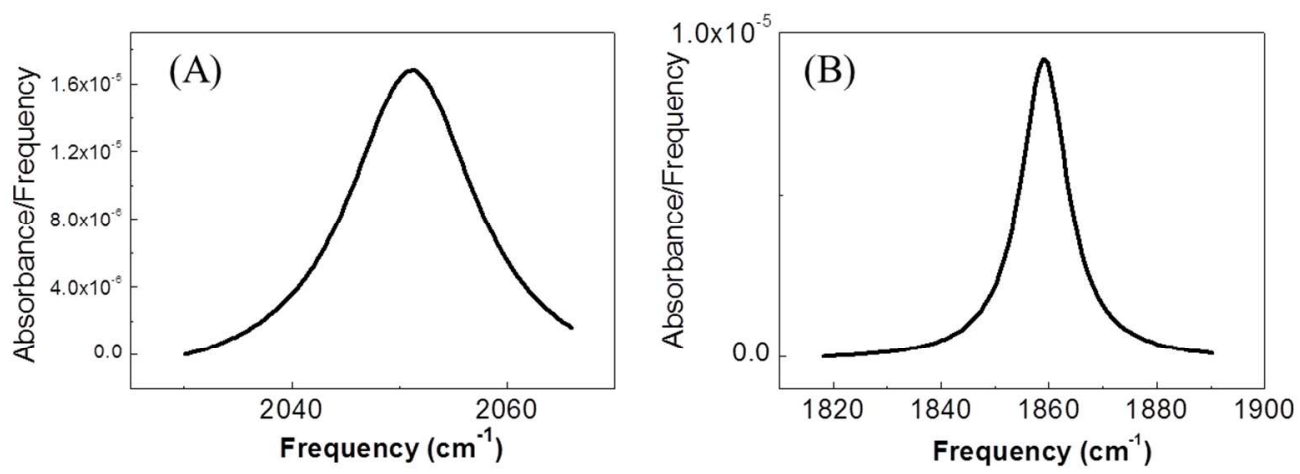

Figure S2 FITR of atop (A) and bridge (B) CO on the $1 \mathrm{~nm}$ Pt CO solution. 


\section{Dephasing rate of Atop CO}

The lineshape fitting is used to obtain the dephasing rate based on the reported method ${ }^{3}$. As shown in Figure S3, the linewidth is around $14.4 \mathrm{~cm}^{-1}$. Thus, the dephasing time $\tau$ is determined as following:

$$
\tau=\frac{1}{2 \pi} \times \frac{100}{14.4 \times 3} p s=0.37 p s \quad \text { Eq. S10 }
$$

This rate is the limit for the energy transfer rate based on the dephasing mechanism ${ }^{4}$.

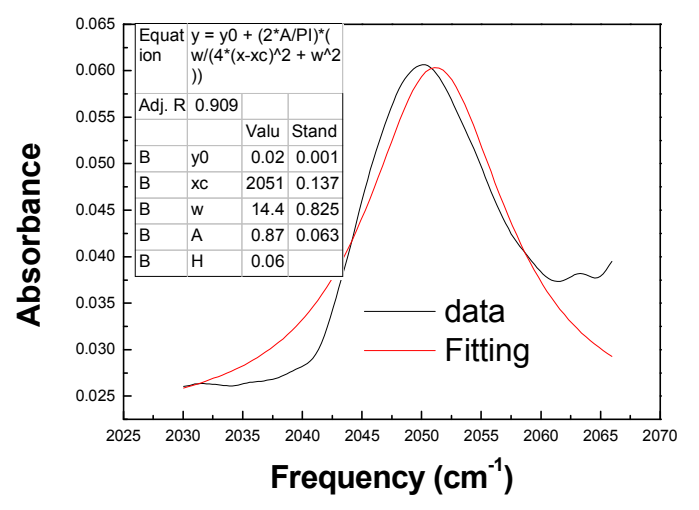

Figure S3 Lorentz fitting of atop CO on the $1 \mathrm{~nm}$ Pt CO solution. 


\section{Energy transfer rate calculation}

Based on the coordination of the $\mathrm{Pt}_{15}$ molecule, the coupling between $\mathrm{CO}_{\mathrm{Atop} 11}$ and $\mathrm{CO}_{\mathrm{Bridge} 11}$ could be calculated by given certain distance $(3.45 \AA)$ and angle $(\kappa=0.86)$. The transition dipole moments of both modes are calculated above.

$$
\beta=\frac{1}{4 \pi \varepsilon_{0} n^{2}} \frac{\mu_{D} \mu_{A} \kappa}{r_{D A}^{3}}=\frac{0.36 \times 0.26 \times\left(3.33 \times 10^{-30}\right)^{2} \times 0.86}{4 \pi \times 8.85 \times 10^{-12} \times 1.43 \times 1.43 \times\left(3.45 \times 10^{-10}\right)^{3}} \times \frac{10^{-2}}{6.63 \times 10^{-34} \times 3 \times 10^{8}}=4.8 \mathrm{~cm}^{-1}
$$

Eq. S11.

The calculated coupling was then used to calculate the energy transfer rate from $\mathrm{CO}_{\text {Atop11 }}$ to $\mathrm{CO}_{\text {Bridgel1 }}$ with dephasing time $(0.37 \mathrm{ps})$ and energy mismatch $\left(187 \mathrm{~cm}^{-1}\right)$.

$$
k_{D A}=\frac{2}{1+e^{\frac{-\Delta \omega}{k T}}} \beta^{2} \frac{\frac{1}{\tau}}{(\Delta \omega)^{2}+4 \beta^{2}+\tau^{-2}}=\frac{2}{1+\exp (-187 / 200)} 4.8^{2} \frac{\frac{1}{\tau}}{187^{2}+4.8^{2}+14.4^{2}}=1 / 395\left(\mathrm{ps}^{-1}\right)
$$




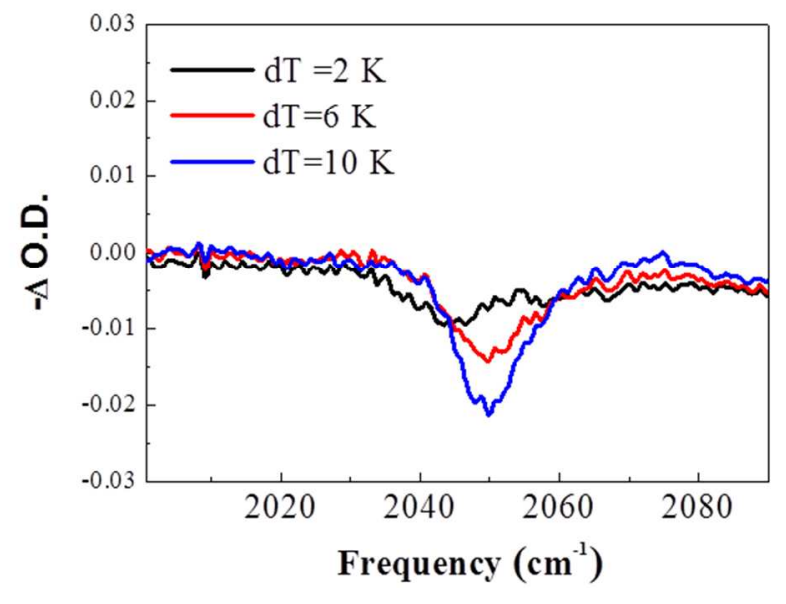

Figure S4 Temperature dependence of CO step site Absorption difference.

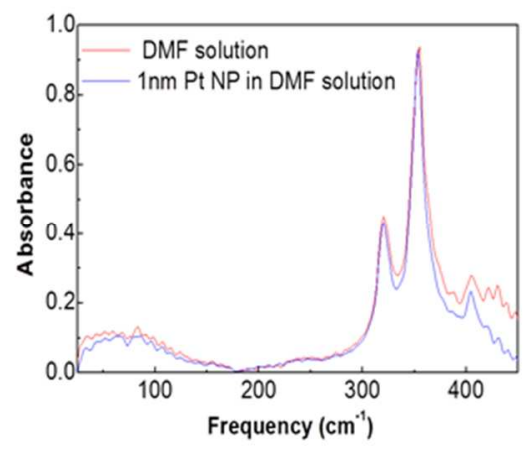

Figure S5 THz absorption spectrum of pure DMF solution and 1nm Pt nanoparticle solution 
Calculated energy transfer rates between each $\mathrm{CO}$ pairs

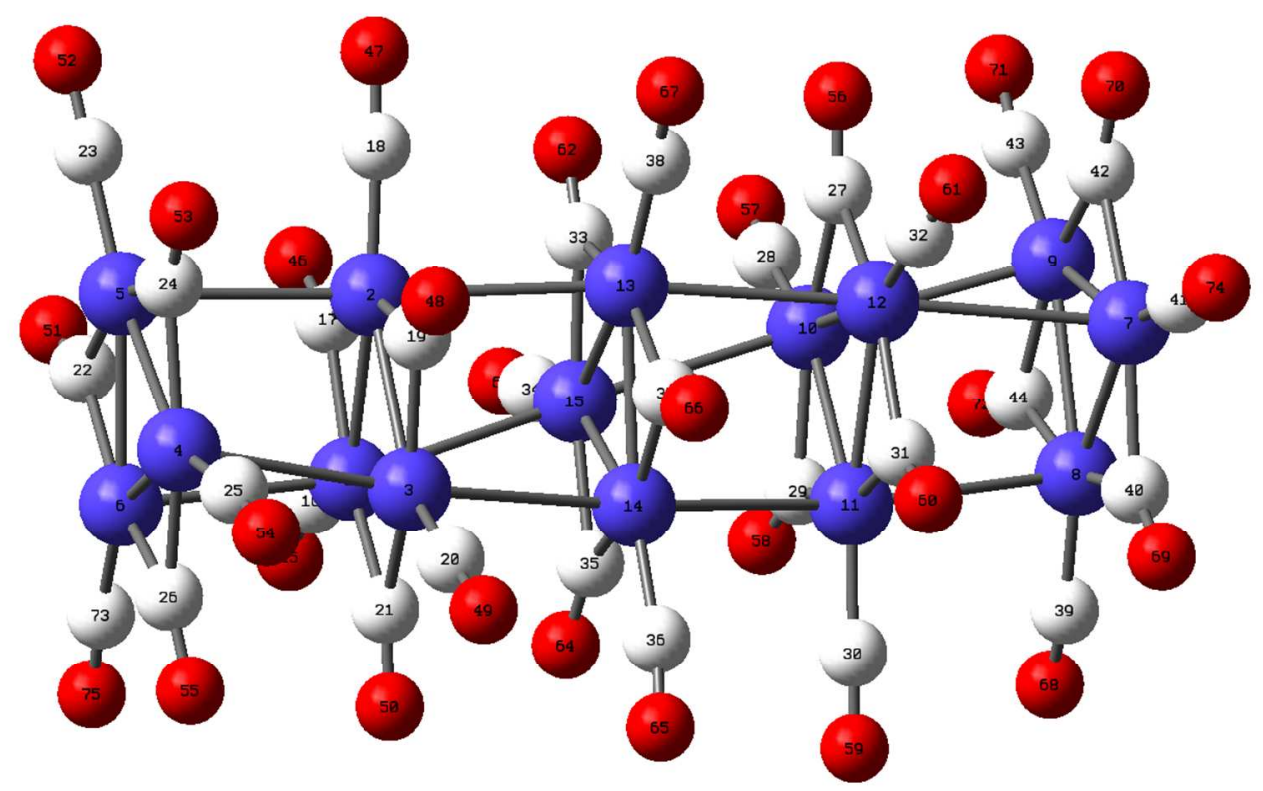

Figure S6 Atom labeled CO and Pt on this cluster structure. 
Table S1 Calculated energy transfer rates ratio compared with $k_{\text {Atop11-Bridgel1 }}$

\begin{tabular}{|c|c|c|c|c|c|c|c|}
\hline $\begin{array}{l}\text { Donor } \\
C O\end{array}$ & Acceptor CO & $\begin{array}{c}\text { Distance }\left(r_{D A}\right) \\
(A)\end{array}$ & $\begin{array}{c}\text { Angle } \\
\text { Value (к) }\end{array}$ & $\begin{array}{c}\text { Rate Ratio } \\
\left(r_{D A}{ }^{6}\right)\end{array}$ & $\begin{array}{l}\text { Rate } \\
\text { Ratio } \\
\left(r_{D A}{ }^{5}\right)\end{array}$ & $\begin{array}{l}\text { Rate } \\
\text { Ratio } \\
\left({r_{D A}}^{4}\right)\end{array}$ & $\begin{array}{l}\text { Rate } \\
\text { Ratio } \\
\left(r_{D A}{ }^{3}\right)\end{array}$ \\
\hline \multirow{7}{*}{$\begin{array}{l}C_{23^{-}} \\
O_{52}\left(\text { Atop }{ }_{11}\right) \\
\text { First layer }\end{array}$} & $\begin{array}{c}\mathrm{C}_{22}-\mathrm{O}_{51} \\
\left(\text { Bridge }{ }_{11}\right)\end{array}$ & 3.45 & 0.86 & 1.00 & 1.00 & 1.00 & 1.00 \\
\hline & $C_{24}-O_{53}$ & 3.5 & 1.05 & 1.33 & 1.36 & 1.38 & 1.40 \\
\hline & $C_{26^{-}} O_{55}$ & 6.9 & 1.99 & 0.10 & 0.19 & 0.37 & 0.71 \\
\hline & $\mathrm{C}_{17}-\mathrm{O}_{46}$ & 4.4 & 0.96 & 0.14 & 0.18 & 0.23 & 0.30 \\
\hline & $C_{19^{-}} O_{48}$ & 5.5 & 0.86 & 0.06 & 0.09 & 0.15 & 0.24 \\
\hline & $C_{21}-O_{50}$ & 7.67 & 1.05 & 0.03 & 0.05 & 0.12 & 0.27 \\
\hline & $C_{33^{-}} O_{62}$ & 6.65 & 0.91 & 0.02 & 0.04 & 0.08 & 0.15 \\
\hline \multirow{6}{*}{$\begin{array}{c}C_{18^{-}} \\
O_{47}(\text { Atop }\end{array}$} & $\mathrm{C}_{22}-\mathrm{O}_{51}$ & 5.1 & 0.90 & 0.10 & 0.15 & 0.22 & 0.33 \\
\hline & $\mathrm{C}_{24} \mathrm{O}_{53}$ & 4.2 & 0.78 & 0.25 & 0.30 & 0.37 & 0.45 \\
\hline & $C_{26^{-}} O_{55}$ & 7.2 & 1.40 & 0.03 & 0.07 & 0.14 & 0.28 \\
\hline & $\mathrm{C}_{17}-\mathrm{O}_{46}$ & 3.5 & 1.00 & 1.17 & 1.19 & 1.22 & 1.24 \\
\hline & $C_{19^{-}} O_{48}$ & 3.6 & 1.13 & 1.42 & 1.46 & 1.50 & 1.55 \\
\hline & $\mathrm{C}_{21^{-}} \mathrm{O}_{50}$ & 6.8 & 1.99 & 0.09 & 0.18 & 0.35 & 0.68 \\
\hline \multirow{2}{*}{$\begin{array}{l}\text { 21) } \\
\text { Second }\end{array}$} & $\mathrm{C}_{33^{-}} \mathrm{O}_{62}$ & 3.8 & 0.88 & 0.57 & 0.63 & 0.70 & 0.77 \\
\hline & $C_{35^{-}} O_{64}$ & 7.1 & 1.28 & 0.03 & 0.06 & 0.12 & 0.25 \\
\hline Layer & $C_{37}-O_{66}$ & 5.3 & 0.97 & 0.09 & 0.15 & 0.22 & 0.34 \\
\hline \multirow{3}{*}{$\begin{array}{c}C_{38^{-}} \\
O_{67}(\text { Atop }\end{array}$} & $\mathrm{C}_{27}-\mathrm{O}_{56}$ & 5.1 & 0.90 & 0.10 & 0.15 & 0.22 & 0.33 \\
\hline & $C_{31}-O_{60}$ & 4.2 & 0.78 & 0.25 & 0.30 & 0.37 & 0.45 \\
\hline & $C_{29}-O_{58}$ & 7.2 & 1.40 & 0.03 & 0.06 & 0.14 & 0.28 \\
\hline
\end{tabular}




\begin{tabular}{|c|c|c|c|c|c|c|c|}
\hline \multirow{3}{*}{ Third } & $C_{17}-O_{46}$ & 5.7 & 0.90 & 0.05 & 0.08 & 0.14 & 0.24 \\
\cline { 2 - 8 } & $C_{19^{-}} O_{48}$ & 4.0 & 0.90 & 0.44 & 0.51 & 0.59 & 0.69 \\
\cline { 2 - 8 } & $C_{21^{-}} O_{50}$ & 7.3 & 1.30 & 0.02 & 0.05 & 0.11 & 0.24 \\
\cline { 2 - 8 } & $C_{33^{-}} O_{62}$ & 3.7 & 0.95 & 0.85 & 0.90 & 0.95 & 1.01 \\
\cline { 2 - 8 } & $C_{35^{-}} O_{64}$ & 6.8 & 1.90 & 0.08 & 0.16 & 0.32 & 0.62 \\
\cline { 2 - 8 } & $C_{37^{-}} O_{66}$ & 3.4 & 1.18 & 2.01 & 1.98 & 1.95 & 1.92 \\
\hline
\end{tabular}




\section{Atomic coordination of this Pt nanocluster with $\mathrm{CO}$ on surface}

The atomic structure of this paper is using the reported structural coordination ${ }^{5}$ of $\mathrm{Pt}_{15} \mathrm{CO}_{30}$ in $\mathrm{C}_{69} \mathrm{H}_{43} \mathrm{~N}_{9} \mathrm{O}_{33} \mathrm{Pt}_{15} \mathrm{Ru}$ with modification of $\mathrm{Pt}-\mathrm{C}$ bond distance and $\mathrm{C}-\mathrm{O}$ bond distance with reported values ${ }^{6}$. The following table $\mathrm{S} 2$ is coordination.

Table S2 $\mathrm{Pt}_{15} \mathrm{CO}_{30}$ coordination

\begin{tabular}{|c|c|c|c|}
\hline Atom & $\mathbf{x}$ & $\mathbf{y}$ & $\mathbf{Z}$ \\
\hline $\mathrm{Pt}$ & 11.41611 & 9.23584 & 9.37992 \\
\hline $\mathrm{Pt}$ & 11.48251 & 8.55184 & 6.81029 \\
\hline $\mathrm{Pt}$ & 11.57373 & 6.67242 & 8.68809 \\
\hline $\mathrm{Pt}$ & 14.58228 & 6.5803 & 7.94639 \\
\hline $\mathrm{Pt}$ & 14.55726 & 8.93195 & 6.70352 \\
\hline $\mathrm{Pt}$ & 14.48772 & 8.8177 & 9.36016 \\
\hline $\mathrm{Pt}$ & 2.35249 & 6.90287 & 7.28624 \\
\hline $\mathrm{Pt}$ & 2.47718 & 8.19267 & 9.60927 \\
\hline $\mathrm{Pt}$ & 2.39182 & 9.56654 & 7.33314 \\
\hline $\mathrm{Pt}$ & 5.38768 & 9.86139 & 8.07333 \\
\hline $\mathrm{Pt}$ & 5.53545 & 7.6951 & 9.62548 \\
\hline $\mathrm{Pt}$ & 5.39527 & 7.43602 & 6.98184 \\
\hline $\mathrm{Pt}$ & 8.44286 & 7.93632 & 6.71632 \\
\hline $\mathrm{Pt}$ & 8.54692 & 7.06496 & 9.22972 \\
\hline $\mathrm{Pt}$ & 8.41014 & 9.67537 & 8.736 \\
\hline $\mathrm{C}$ & 11.31326 & 10.8497 & 10.1704 \\
\hline $\mathrm{C}$ & 11.36709 & 10.36174 & 7.70351 \\
\hline $\mathrm{C}$ & 11.36548 & 8.90029 & 5.04822 \\
\hline
\end{tabular}




\begin{tabular}{|c|c|c|c|}
\hline $\mathrm{C}$ & 11.6048 & 6.53919 & 6.68907 \\
\hline $\mathrm{C}$ & \begin{tabular}{|l|}
11.65045 \\
\end{tabular} & 4.90377 & 9.01369 \\
\hline $\mathrm{C}$ & \begin{tabular}{|l|l}
11.50315 \\
\end{tabular} & 7.55892 & 10.5018 \\
\hline $\mathrm{C}$ & \begin{tabular}{|l|}
14.48263 \\
\end{tabular} & 10.36767 & 8.12251 \\
\hline $\mathrm{C}$ & \begin{tabular}{|l|}
14.69426 \\
\end{tabular} & 9.90046 & 5.19248 \\
\hline $\mathrm{C}$ & \begin{tabular}{|l|}
14.62364 \\
\end{tabular} & 7.06298 & 5.98534 \\
\hline $\mathrm{C}$ & 14.50941 & 4.78255 & 7.89389 \\
\hline $\mathrm{C}$ & 14.5205 & 6.89782 & 9.92143 \\
\hline $\mathrm{C}$ & 5.29666 & 9.27179 & 6.14343 \\
\hline $\mathrm{C}$ & 5.31835 & 11.6257 & 7.72347 \\
\hline $\mathrm{C}$ & 5.50511 & 9.66894 & 10.08071 \\
\hline $\mathrm{C}$ & 5.6468 & 7.10324 & 11.32174 \\
\hline $\mathrm{C}$ & 5.51617 & 6.05227 & 8.44848 \\
\hline $\mathrm{C}$ & 5.36077 & 6.15678 & 5.71601 \\
\hline $\mathrm{C}$ & 8.34752 & 9.94504 & 6.73506 \\
\hline $\mathrm{C}$ & 8.33646 & 11.42885 & 9.13584 \\
\hline $\mathrm{C}$ & 8.50215 & 8.6541 & 10.47694 \\
\hline $\mathrm{C}$ & 8.63863 & 5.82109 & 10.53192 \\
\hline $\mathrm{C}$ & 8.55086 & 6.06677 & 7.47364 \\
\hline $\mathrm{C}$ & \begin{tabular}{|l|}
8.30419 \\
\end{tabular} & 7.44572 & 4.98346 \\
\hline $\mathrm{C}$ & 2.49845 & 8.36908 & 11.40048 \\
\hline $\mathrm{C}$ & 2.43089 & 6.24289 & 9.19378 \\
\hline $\mathrm{C}$ & 2.29321 & 5.29219 & 6.48486 \\
\hline $\mathrm{C}$ & 2.30291 & 8.26188 & 5.79354 \\
\hline $\mathrm{C}$ & 2.39182 & \begin{tabular}{|l|}
11.05942 \\
\end{tabular} & 6.32749 \\
\hline
\end{tabular}




\begin{tabular}{|c|c|c|c|}
\hline $\mathrm{C}$ & 2.53921 & 10.17987 & 9.25211 \\
\hline $\mathrm{O}$ & \begin{tabular}{|l|}
11.32423 \\
\end{tabular} & 11.51686 & 11.10703 \\
\hline $\mathrm{O}$ & \begin{tabular}{|l|}
11.37689 \\
\end{tabular} & 11.4724 & 7.30507 \\
\hline $\mathrm{O}$ & \begin{tabular}{|l|}
11.29772 \\
\end{tabular} & 9.291 & 3.96875 \\
\hline $\mathrm{O}$ & \begin{tabular}{|l|}
11.71806 \\
\end{tabular} & 5.62359 & 5.95338 \\
\hline $\mathrm{O}$ & \begin{tabular}{|l|}
11.60418 \\
\end{tabular} & 3.79335 & 9.30917 \\
\hline $\mathrm{O}$ & 11.51041 & 7.238 & 11.6373 \\
\hline $\mathrm{O}$ & 14.45661 & 11.54094 & 7.9994 \\
\hline $\mathrm{O}$ & \begin{tabular}{|l|}
14.78178 \\
\end{tabular} & 10.51922 & 4.2271 \\
\hline $\mathrm{O}$ & 14.66584 & 6.51301 & 4.94219 \\
\hline $\mathrm{O}$ & \begin{tabular}{|l|}
14.48351 \\
\end{tabular} & 3.64795 & 8.07969 \\
\hline $\mathrm{O}$ & \begin{tabular}{|l|}
14.42203 \\
\end{tabular} & 6.28734 & 10.92643 \\
\hline $\mathrm{O}$ & 5.22704 & 9.59248 & 5.05787 \\
\hline $\mathrm{O}$ & 5.10882 & 12.83726 & 7.5285 \\
\hline $\mathrm{O}$ & 5.67208 & 10.4271 & 10.95005 \\
\hline $\mathrm{O}$ & 5.71794 & 6.72511 & 12.40547 \\
\hline $\mathrm{O}$ & 5.56169 & 4.89591 & 8.67902 \\
\hline $\mathrm{O}$ & 5.21156 & 5.43035 & 4.92322 \\
\hline $\mathrm{O}$ & 8.23581 & 10.7757 & 5.90409 \\
\hline $\mathrm{O}$ & 8.31385 & 12.54788 & \begin{tabular}{|l|l|}
9.39996 \\
\end{tabular} \\
\hline $\mathrm{O}$ & 8.65775 & 8.97084 & 11.60294 \\
\hline $\mathrm{O}$ & 8.80689 & 5.09548 & 11.40809 \\
\hline $\mathrm{O}$ & 8.60219 & 4.9006 & 7.30101 \\
\hline $\mathrm{O}$ & 8.33418 & 6.95483 & 3.94393 \\
\hline $\mathrm{O}$ & \begin{tabular}{|l|}
2.4999 \\
\end{tabular} & 8.78667 & 12.4795 \\
\hline
\end{tabular}




\begin{tabular}{|l|l|l|l|}
\hline $\mathrm{O}$ & 2.43982 & 5.17626 & 9.71238 \\
\hline $\mathrm{O}$ & 2.24628 & 8.01422 & 4.63589 \\
\hline $\mathrm{O}$ & 2.29582 & 12.01242 & 5.68749 \\
\hline $\mathrm{O}$ & 2.61309 & 11.28017 & 9.67992 \\
\hline $\mathrm{C}$ & 14.42449 & 9.55801 & 10.99965 \\
\hline $\mathrm{O}$ & 2.21521 & 4.24481 & 6.01049 \\
\hline $\mathrm{O}$ & 14.38657 & 9.86739 & 12.1066 \\
\hline
\end{tabular}

\section{References:}

1. Bian, H. T.; Wen, X. W.; Li, J. B.; Zheng, J. R., Mode-specific intermolecular vibrational energy transfer. II. Deuterated water and potassium selenocyanate mixture. J Chem Phys 2010, $133(3), 034505$.

2. Knox, R. S.; van Amerongen, H., Refractive index dependence of the Förster resonance excitation transfer rate. $J$ Phys Chem B 2002, 106 (20), 5289-5293.

3. Chen, H.; Wen, X.; Li, J.; Zheng, J., Molecular Distances Determined with Resonant Vibrational Energy Transfers. J. Phys. Chem. A 2014, 118 (13), 2463-2469.

4. $\quad$ Chen, H.; Wen, X.; Guo, X.; Zheng, J., Intermolecular Vibrational Energy Transfers in Liquids and Solids. Phys Chem Chem Phys 2014, 16, 13995-14014.

5. Femoni, C.; Iapalucci, M. C.; Longoni, G.; Lovato, T.; Stagni, S.; Zacchini, S., SelfAssembly of [Pt3 n (CO) $6 \mathrm{n}$ ] 2-(n= 4- 8) Carbonyl Clusters: from Molecules to Conducting Molecular Metal Wires. Inorg Chem 2010, 49 (13), 5992-6004.

6. Longoni, G.; Chini, P., Synthesis and chemical characterization of platinum carbonyl dianions [Pt3 (CO) 6] n2-(n=. apprx. 10, 6, 5, 4, 3, 2, 1). A new series of inorganic oligomers. $J$ Am Chem Soc 1976, 98 (23), 7225-7231. 